\title{
The cost of shifting attention in depth between real and mirrored space
}

Kimura, T. 1), Shinohara, K. 2), Kitamura, A. 2), and Kinosada, Y. ${ }^{3}$ )

1) Kansai University of Welfare Sciences, Osaka, Japan (takimura@tamateyama.ac.jp)

2) Graduate School of Human Sciences, Osaka University, Osaka, Japan

\section{Background}

Although mirrors are commonly used, how the human visual system operates in mirrored space remains unclear. Humans are capable of acquiring necessary information in mirrored space, even though all the images are reversed in reference to objects in real space.

Therefore, in the present study, two experiments were conducted under real and mirrored observation conditions to compare the characteristics associated with shifting attention in depth.
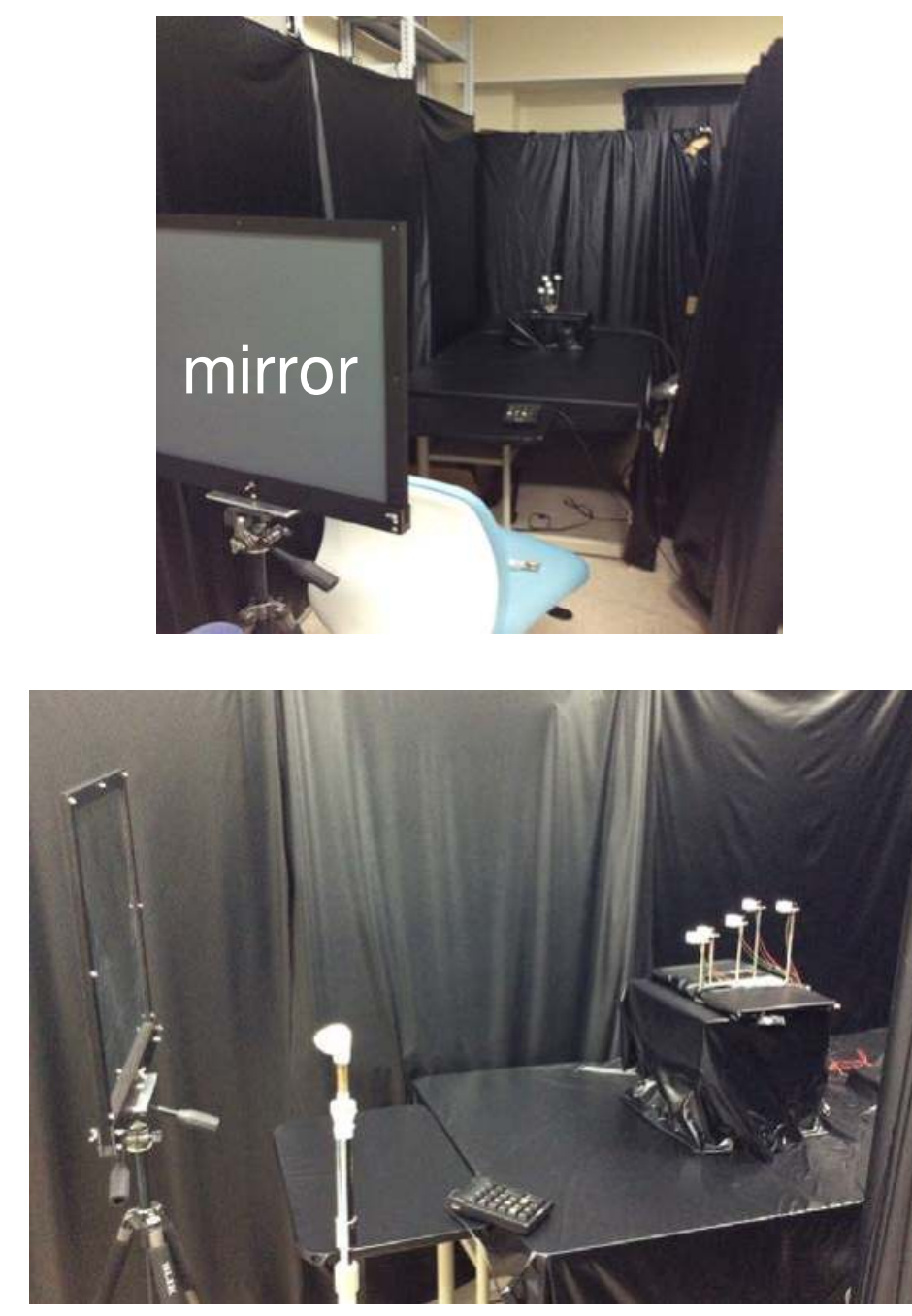

Figure1 The apparatus of this experiment. A fixation point was located $120 \mathrm{~cm}$ from the participants. Targets were located $40 \mathrm{~cm}, 80 \mathrm{~cm}$ (near), $160 \mathrm{~cm}$, and $200 \mathrm{~cm}$ (far) from the participants.

\section{Methods}

Participants:

The study participants were 12 observers (5 male and 7 female, mean age: 24 years old, $S D=2.55$ ). All participants reported normal or corrected to normal vision and provided informed consent.

They observed the stimuli using binocular vision.

\section{Apparatus and Stimuli:}

A fixation point was located $120 \mathrm{~cm}$ from the participants. Four cubes with LEDs were arranged nearer to and farther from the fixation point under different depth conditions (Figure 1).

The cueing paradigm (Posner et al., 1980) was used to clarify how well the observers could control their attention distribution. Information about the direction of a target relative to the fixation point (near/far) was based on color.

Observers who used a chin rest were required to detect the target depending on pre-cues.

Procedure:

The duration of the cue presentations was $300 \mathrm{~ms}$. After the disappearance of the cue, a single target was presented (Figure. 2).

Under the real condition, the stimuli were presented in front of the observer, whereas, under the mirror condition, the stimuli were presented through a mirror $(43 \times 45 \mathrm{~cm})$ behind the observer.

\section{Experimental design:}

The experimental design consisted of two observation conditions (real and mirrored), two types of cue validity (valid and invalid), and four target locations.

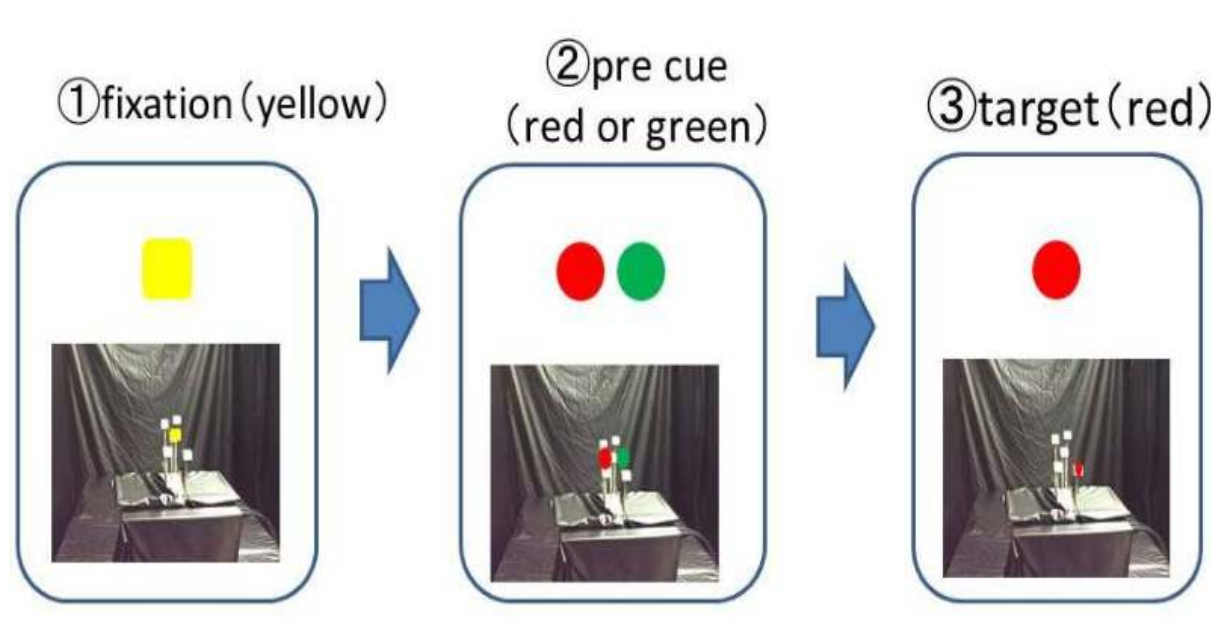

Figure 2 The procedure of this experiment

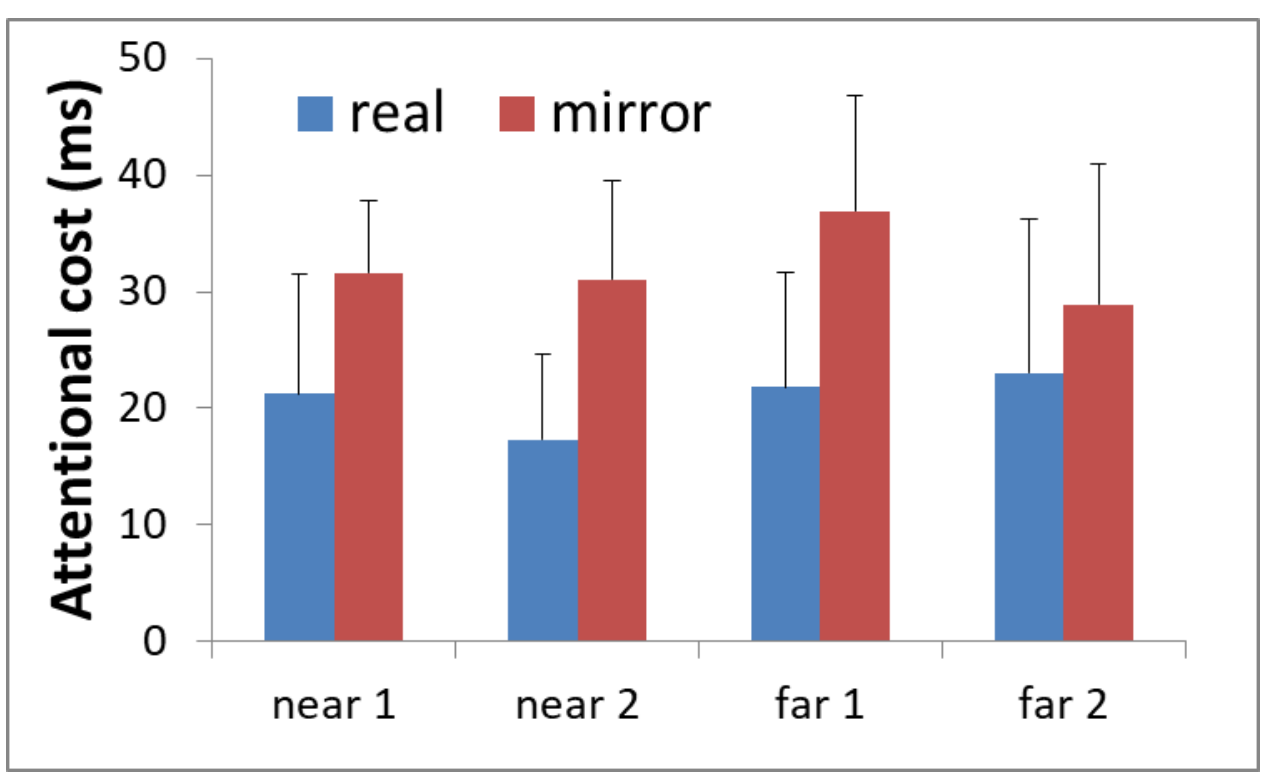

Figure 3 Attentional cost in real and mirrored conditions. Error bars indicate standard error (SE)

\section{Results and Discussion}

The attentional costs were calculated (invalid trials minus valid trials) for each target location in the real and mirrored space. Attentional cost means the time to reallocate opposite side of fixation poin (e.g., "from near space to far space").

The results of a two-way ANOVA (observation $\times$ target locations) indicated a marginal difference in the main effect of the observation condition $[F(1,11)=3.44$ $\mathrm{p}=0.09]$; however, no difference was observed in the main effect of target location, and no significant interaction was identified.

Therefore, the attentional cost in mirrored space was larger than that in real space (32 vs. $21 \mathrm{~ms}$, respectively).

The findings of the present study sugges that the cost for reallocating attention in terms of depth might be effortless under real observation conditions.

Therefore, the difficulty in allocating attention to the rear space might represent a particular characteristic of mirrored space.

\section{References}

Posner, M.I., Snyder, C.R.R., and Davidson, B.J. (1980). Attention and detection of signals Journal of Experimental Psychology: General, 109, 160-174.

This study was supported by Japan Society for the Promotion of Sciences (KAKENHI No.24730633) 